\title{
Consumo de Bebidas Alcohólicas y factores relacionados en Estudiantes de Odontología
}

\author{
Luisa Leonor Arévalo Tovara ${ }^{a}$ Antonio José Díaz Caballero ${ }^{\text {, }}$ \\ Farith Damián González Martínez ${ }^{c}$, Miguel Angel Simancas Pallares ${ }^{d}$
}

a Odontóloga. Especialista en Periodoncia. Decana Facultad de Odontología. Profesor Titular Universidad de Cartagena.

b Odontólogo. Estudiante de Doctorado en Ciencias Biomédicas. Profesor titular Universidad de Cartagena.

c Odontólogo. Magíster en Salud Pública. Profesor Auxiliar Departamento de Investigación. Facultad de Odontología Universidad de Cartagena.

${ }^{d}$ Odontólogo. Estudiante de Maestría en Epidemiología Clínica. Universidad Nacional de Colombia.

Correspondencia: Farito Damián González Martínez, Departamento de Investigaciones, Universidad de Cartagena, Facultad de Odontología, Campus de la Salud Barrio Zaragocilla, Cartagena de Indias, Bolívar, Colombia. Tel 5756698172 ext. 110, correo electrónico: farithgm@hotmail.com.

Recibido el 30 de diciembre de 2009 .

Aceptado para su publicación el 30 de marzo de 2010.

\section{RESUMEN}

Objetivo. Describir el consumo de bebidas alcohólicas entre estudiantes de Odontología de una universidad pública y evaluar su relación con variables sociodemográficas.

Diseño. Estudio observacional de corte transversal.

Emplazamiento. Municipio de Cartagena de Indias, Colombia.

Participantes. Se obtuvo una muestra de 153 estudiantes que cursaban entre primero y décimo semestre, a través de un muestreo probabilístico proporcional al tamaño de cada grado.

Mediciones principales. Se completó un cuestionario estructurado con las siguientes variables: consumo de bebidas alcohólicas, preferencias por el tipo de bebida, grado de bebedor, motivos del consumo, consecuencias y afectación de la integridad física. El análisis se realizó en el programa STATA X versión para Windows 10.0, a partir de odds ratios con intervalos de confianza del 95\% y un análisis multivariable a través de regresión logística. Resultados. A partir de 153 participantes, se obtuvo una prevalencia del consumo de alcohol del $86,7 \%$, siendo mas frecuente el consumo los fines de semana $(55,5 \%)$ y la consecuencia más frecuente fue el deterioro de las relaciones familiares en el 47,8 \%. En cuanto a las relaciones con variables sociodemográficas, al ajustar por edad, las de mayor fuerza fueron la procedencia y el género. Al ajustar por género, los estudiantes de zonas urbanas tuvieron cinco veces mayor probabilidad de consumo.

Conclusiones. Los estudiantes de odontología de esta población presentan un consumo alto de bebidas alcohólicas, introduciendo esté hábito en su realidad y utilizándolo como mediador social en los eventos estresantes que la rodean.

Palabras clave. Consumo de Bebidas Alcohólicas, Salud Pública, Estudiantes Universitarios.

\section{ABSTRACT}

Alcohol consumption and related factors in dental students

Objective. Describe alcohol consumption among dental students and to evaluate its relationship with sociodemographic variables.

Design. Cross-sectional observational study.

Setting. The town of Cartagena de Indias - Colombia.

Participants. We obtained a sample of 153 dental students between first and tenth semester, through a probabilistic sampling proportional to the number of individuals in each grade.

Main measurements. Was used a structured survey with the following variables, alcohol consumption, frequency and preferences for the type of drink, beverage choice, reasons for consumption, consequences and disruption of physical integrity. The statistical analysis was performed in STATA X ® program for Windows version 10.0, from odds ratios with confidence levels of $95 \%$ and multivariate analysis through logistic regression. Results. With 153 participants, we obtained a prevalence of alcohol consumption was $86.7 \%$, with more frequent consumption on weekends $(55.5 \%)$ and the most frequent consequences of alcohol drinkers was the deterioration of family relationships with $47.8 \%$. With regard to relations with sociodemographic variables, adjusting for age, the largest associations were the urban or rural origin and gender. After adjusting for gender, urban students were five times more probablility of consumption.

Conclusions. Dental students in this survey consume with high frequency alcoholic beverages, bringing this to its reality and using it as a conductive in the stress moments.

Key words. Alcohol Drinking, Public Health, College Students. 


\section{INTRODUCCIÓN}

Los conflictos relacionados con el alcohol se encuentran entre los más apremiantes en América Latina y el mundo. El consumo por encima de los niveles permisibles se asocia no sólo a cifras importantes de morbilidad y mortalidad, sino también a muchos problemas sociales. Sumado a esto, tiene graves efectos adversos en las familias y la comunidad $^{1}$. El problema radica en que la mayoría de los individuos cree que, mientras no se conviertan en alcohólicos típicos, la consecuencia de beber frecuentemente y en altas dosis no son alarmantes, sin embargo, los efectos del alcohol en la salud corporal pueden tornarse graves e irreversibles.

La clasificación del paciente que reúne criterios para dependencia de alcohol está ligada a la línea que separa la normalidad de la enfermedad y se encuentra entre el bebedor social y el bebedor abusivo sin dependencia ${ }^{2-6}$. Como bebedor social se encuentra el sujeto que ingiere alcohol sin transgredir las normas sociales, pues el alcohol no le produce efectos biológicos y psicosociales nocivos, manteniendo su libertad ante esta sustancia y presentando entre ninguno y cuatro estados de embriaguez ligeros ó fuertes al año. El bebedor moderado es aquel que consume alcohol hasta tres veces a la semana en cantidades menores que un cuarto de botella de ron, una botella de vino o cinco botellas de cerveza de baja graduación, hasta menos de doce estados de embriaguez ligera al año. El bebedor excesivo, dependiente alcohólico, además de lo anterior presenta complicaciones psíquicas y somáticas y sus estados de embriaguez ligera ó fuerte son más de doce veces al año $0^{7,8}$.
Dentro de la comunidad estudiantil odontológica se exponen comportamientos sociales relacionados con el consumo de alcohol que pueden variar dependiendo del semestre y las obligaciones académicas. En este sentido, el objetivo de esta investigación fue describir el consumo de bebidas alcohólicas entre estudiantes de odontología de una universidad pública de la ciudad de Cartagena, Colombia, y evaluar su relación con variables sociodemográficas.

\section{MATERIAL Y MÉTODO}

Este estudio fue de tipo observacional de corte transversal. La muestra se calculó a partir de un nivel de confianza del $95 \%$, un error permisible del 5 $\%$ y una frecuencia esperada del evento del $89,0 \%{ }^{5}$, obteniéndose 153 estudiantes. Para la selección se utilizó un muestreo estratificado proporcional al tamaño de cada semestre, teniendo en cuenta que fueran estudiantes universitarios matriculados en el programa de odontología de la Universidad de Cartagena durante el segundo semestre de 2007, que aceptaran elaborar un cuestionario tipo encuesta y lo confirmaran a través de un consentimiento informado por escrito. A pesar de que esta investigación en seres humanos no tiene ningún riesgo para la salud, como lo dispone la resolución 008430 de 1993 del Ministerio de Salud de Colombia ${ }^{9}$, puede constituir una violación a la intimidad de los sujetos involucrados, ya que el alcohol se concibe con un hábito muy difícil de reconocer por los actores involucrados, sobre todo al tener en cuenta que el medio en el cual están involucrados los sujetos de estudio está compuesto por profesionales de la sa-

\begin{tabular}{lccc}
\hline & $\begin{array}{c}\text { Número } \\
\mathbf{n = 1 5 3}\end{array}$ & $\begin{array}{c}\text { Prevalencia } \\
(\%)^{*}\end{array}$ & IC 95\% \\
\hline Consumo Alcohol & 129 & 86,7 & $80,8-92,2$ \\
SI & 24 & 13,2 & $7,3-19,1$ \\
No & 27 & 21,5 & $13,7-29,3$ \\
Frecuencia consumo & 13 & 9,6 & $4,2-15,0$ \\
Cualquier día & 89 & 55,4 & $46,4-64,5$ \\
Días de la semana & & & $7,3-19,1$ \\
Fin de semana & 24 & 13,2 & $17,6-33,5$ \\
Frecuencia consumo al mes & 41 & 25,6 & $12,6-27,5$ \\
Una vez & 26 & 20,1 & $10,7-24,1$ \\
Dos veces & 29 & 17,4 & $65,4-81,3$ \\
Tres veces & & & $5,4-16,6$ \\
Más de tres veces & 107 & 73,4 & $0,8-5,2$ \\
Época del año & 20 & 11,1 & 2,1 \\
Cualquiera & 2 & 2,1 & \\
Vacaciones & & & \\
Periodo académico & & & \\
\hline
\end{tabular}

Tabla 1. Consumo de Alcohol en estudiantes de Odontología. * Estimadores ajustados por diseño de muestreo. 
lud con un conocimiento profundo sobre los prejuicios de consumir alcohol y sus consecuencias en el rendimiento académico. Desde este punto de vista se solicitó aprobación por parte del Comité de Ética Institucional.

Para la recogida de los datos sobre el consumo de alcohol y sus características se diseñó un cuestionario tipo encuesta estructurada, que contenía 14 preguntas politómicas y dicotómicas con única respuesta, con el fin de evaluar las siguientes variables: consumo de alcohol, frecuencia y preferencia por el tipo de bebidas, grado de bebedor, motivo y consecuencias del consumo, afectación de la integridad personal, edad, género, estrato socioeconómico, procedencia y semestre. Antes de aplicar el cuestionario se tuvo en cuenta la pertinencia de las preguntas, la coherencia con el objetivo del estudio, la sintaxis y semántica de cada construcción gramatical. Luego, a través de un piloto, se puso a prueba el instrumento a un grupo de 50 estudiantes universitarios con características similares para valorar si realmente las preguntas medían las variables objeto de estudio y si las puntuaciones obtenidas eran confiables.

La información recogida se tabuló en una matriz del programa Microsoft Excel 2007, y luego fueron analizados usando el programa STATA X versión para Windows 10.0. Las variables cualitativas se analizaron por proporciones con intervalos de confianza del $95 \%$ y las variables cuantitativas a partir de las medidas de centralización y dispersión. Con el fin de poner a prueba la fuerza de asociación entre va-

\begin{tabular}{lccc}
\hline & $\begin{array}{c}\text { Número } \\
\mathbf{n = 1 5 3}\end{array}$ & $\begin{array}{c}\text { Prevalencia } \\
(\mathbf{( \% )}\end{array}$ & IC 95\% \\
\hline Es un bebedor social & 78 & & $47,0-64,8$ \\
$\mathrm{Si}$ & 51 & 55,9 & $22,6-38,8$ \\
No & & 30,7 & \\
Bebida preferida & 30 & 21,9 & $14,1-29,5$ \\
Cerveza & 24 & 14,2 & $8,2-20,2$ \\
Whisky & 15 & 10,6 & $5,0-16,1$ \\
Ron & 7 & 3,5 & $0,4-6,6$ \\
Cócteles & 53 & 36,4 & $27,6-45,2$ \\
Cualquier tipo & 5
\end{tabular}

Tabla 2. Tipo de bebida preferida y frecuencia de tomador social. * Estimadores ajustados por diseño de muestreo.

\begin{tabular}{lccc}
\hline & $\begin{array}{c}\text { Número } \\
\mathbf{n = 1 5 3}\end{array}$ & $\begin{array}{c}\text { Prevalencia } \\
(\mathbf{\%})^{*}\end{array}$ & IC 95\% \\
\hline Motivos del consumo & & & $15,6-31,0$ \\
Sentirse bien & 32 & 23,3 & $6,3-17,6$ \\
Hacer amigos & 20 & 12,0 & $1,7-11,3$ \\
Vínculos familiares & 7 & 6,5 & $0,1-6,3$ \\
Moda & 5 & 3,2 & $32,9-50,8$ \\
Ninguno & 65 & 41,8 & \\
Consecuencias del consumo & & & $3,9-14,4$ \\
Deterioro de relaciones afectivas & 14 & 9,1 & $14,9-30,7$ \\
Pérdida de objetos personales & 26 & 22,8 & $1,2-9,0$ \\
Venta de objetos personales & 7 & 5,1 & $38,7-56,8$ \\
Deterioro de relaciones familiares & 79 & 47,8 & $7,3-18,1$ \\
Ninguna & 24 & 13,2 & \\
Afectación de la Integridad & & & $8,5-21,8$ \\
Peleas con amigos & 28 & 15,1 & $6,6-18,3$ \\
Sexo inducido & 18 & 12,4 & $8,0-21,2$ \\
Lagunas mentales & 19 & 14,6 & $3,9-14,8$ \\
Accidentes & 11 & 9,4 & $0,2-4,6$ \\
Intoxicación & 4 & 2,2 & $24,4-41,4$ \\
Ninguna & 57 & 32,9 & \\
\hline
\end{tabular}

Tabla 3. Motivos y consecuencias de consumo de alcohol en estudiantes de Odontología. * Estimadores ajustados por diseño de muestreo 
riables, se realizó un analisis bivariado a través de los odds ratios y con el fin de hacer ajustes simultáneos de los estimadores de asociación se realizó un análisis multivariable a través de regresión logística.

\section{RESULTADOS}

El número de participantes que completaron la encuesta fue de 153, con un promedio de edad de $20,3$ (DE $=2,04)$. En cuanto al género, los varones representaron el $40,5 \%$ y las mujeres un 59,5 $\%$. Con respecto a la distribución por semestres, quinto y sexto fueron los cursos que más aportaron participantes con un $13,1 \%$, seguido de cuarto y séptimo con $11,1 \%$, mientras que en los de más baja participación estuvo tercero con $6,5 \%$ y octavo $\operatorname{con} 7,8 \%$.

La prevalencia del consumo de alcohol fue 86,7 $\%$ (IC 95\%; 80,8-92,2), siendo más frecuente el consumo los fines de semana con el $55,4 \%(46,4-$ $64,5)$. Por otra parte, el 25,6 \% $(17,7-33,5)$ de los participantes manifestaron que consumen bebidas alcohólicas con una periodicidad de dos veces al mes. Con respecto a la época del año, el 73,4 \% $(61,5-81,3)$ expresó que en cualquier época consumen bebidas alcohólicas (tabla 1).

En cuanto a los consumidores, el $55,9 \%(47,0-64,8)$ se considera un bebedor social, siendo la cerveza la bebida preferida por el 21,9 \% (14,1-29,5) de los encuestados (tabla 2).

En lo referente a los motivos del consumo, el 23,3 $\%(15,6-31,0)$ de los participantes lo hace para sentirse bien. Por otro lado, el $47,8 \%(38,8-56,8)$ manifiesta que como consecuencia del consumo se ha producido un deterioro de las relaciones familiares y en cuanto a la integridad personal, el 15,1 \% (8,5$21,8)$ reportó que el consumo de alcohol ha influido en la riñas con amigos y al $12,4 \%(6,6-18,3)$ los ha inducido a tener relaciones sexuales (tabla 3 ).

Con respecto al análisis bivariado, entre el consumo de bebidas alcohólicas y variables sociodemográficas como edad, género, estrato y procedencia, sólo se encontró relación estadísticamente significativa con la procedencia, siendo los estudiantes que vienen de poblaciones urbanas los de mayor tendencia al consumo (OR = 3,29; IC 95\%: 1,159,$38 ; p=0.02$ ). En cuanto al análisis multivariable, se ajustaron los estimadores por edad y se obtuvo un modelo bueno ( $\left.p=0.000 ; x^{2}=258\right)$, el cual muestra la fuerza de interacción entre el consumo de alcohol y las variables género, procedencia y semestre. Por otra parte, al ajustar por género, se obtiene un modelo bueno $\left(p=0.002 x^{2}=16,7\right)$, con aumento de la fuerza de asociación inicial para las variables procedencia y semestres (tabla 4).

\section{DISCUSIÓN}

Los resultados del presente estudio pueden ser útiles para hacer un análisis de algunos comportamientos sociales adquiridos durante un proceso educativo y se consideran como una exploración de este problema para la puesta en marcha de programas preventivos de este tipo de conductas, liderados desde la división de Bienestar Universitario de la institución educativa donde se obtuvo la información. Sin embargo, es claro que estos hallazgos solo representan una aproximación a la realidad a partir de las percepciones de los participantes y cuya veracidad depende estrictamente de la voluntad de éstos para elaborar las respuestas con la sinceridad de sus experiencias vividas, a partir de un cuestionario.

Según la información obtenida, en esta población se presenta una alta prevalencia del consumo de bebidas alcohólicas, siendo los fines de semana los días de mayor frecuencia de este consumo, Abreu2 reportó a través de un estudio que el $79,2 \%$ de los estudiantes consumen bebidas alcohólicas, pero de éstos solo el 9,9\% son bebedores moderados, lo que implica un patrón de consumo menos frecuente. Estos resultados se consideran alarmantes, teniendo en cuenta que provienen de una comunidad perteneciente al sector de la salud y que debería promover un estilo de vida saludable.

Por otro lado, para una proporción importante de los sujetos encuestados en el presente estudio, el tipo de bebida preferida fue la cerveza, aunque muchos manifestaron que esta elección es circunstancial, la cual depende de factores diferentes de su voluntad. En este mismo sentido, Guibert ${ }^{10}$ encontró que el tipo de bebidas consumidas con mayor frecuencia en estudiantes universitarios fue la cerveza con $88,2 \%$, observándose, además, que habitualmente consumen alcohol inducidos por sus amigos y por la aceptación familiar. En comparación con estos datos, en el presente estudio los participantes manifestaron que consumen bebidas alcohólicas motivados por la sensación de bienestar, por compartir con los amigos y familiares, situación que refleja un profundo significado psicológico en el contexto académico y social de los estudiantes. De esta forma, se facilita la relación e integración de los individuos, quizás por ser un deleite común. Desde esta perspectiva, Medina ${ }^{11}$, en un estudio en México, revela la importancia del contexto familiar y social en el inicio del consumo y consumo habitual de alcohol entre la población estudiantil, lo que contribuye a que este hábito se mantenga dentro de los grupos poblacionales sin ninguna percepción anormal. Por otra parte, Herrán ${ }^{12}$ identificó que el fin de semana es el tiempo de predilección para los consumidores activos, información que concuerda con los hallaz- 


\begin{tabular}{|c|c|c|c|c|c|c|}
\hline & \multicolumn{6}{|c|}{ Consumo de alcohol } \\
\hline & \multicolumn{2}{|c|}{ Bivariado } & \multicolumn{2}{|c|}{ Multivariado } & \multicolumn{2}{|c|}{ Multivariado $f$} \\
\hline & $\mathrm{OR}^{\mathrm{b}}$ & IC 95\% & $\mathrm{OR}^{\mathrm{c}}$ & IC $95 \%$ & $\mathrm{OR}^{\mathrm{d}}$ & IC $95 \%$ \\
\hline Edad & 0,97 & $0,70-1,34$ & - & - & 1,3 & $0,93-1,77$ \\
\hline Género & 1,42 & $0,47-4,29$ & 2,7 & $2,16-3,43$ & - & - \\
\hline Procedencia & $3,29^{a}$ & $1,15-9,38$ & 3,7 & $2,97-4,58$ & 5,3 & $1,55-17,9$ \\
\hline Estrato & 0,63 & $0,23-1,77$ & 0,9 & $0,71-1,09$ & 0,7 & $0,20-2,16$ \\
\hline Semestre & 0,88 & $0,75-1,03$ & 0,9 & $0,85-0,92$ & 0,7 & $0,54-0,94$ \\
\hline
\end{tabular}

Tabla 4. Relación entre Consumo de Alcohol y variables Sociodemográficas en estudiantes. aP=0.02, bodds ajustados por diseño de muestreo, codds ajustados por edad, dodds ajustados por género, esignificación del modelo de regresión por edad $(p=0.000 ; \chi 2=258)$, fsignificación del modelo de regresión por género $\left(p=0.002 ; x^{2}=16,7\right)$.

gos obtenidos en la población estudiantil encuestada, sin embargo también existe un gran porcentaje de estudiantes $(21,5 \%)$ que ingieren alcohol entre semana, lo que resulta preocupante por la poca discriminación de los períodos en donde se hace necesario mantenerse sobrio para la consecución del desempeño académico adecuado.

En cuanto a la afectación de la integridad personal, el presente estudio arrojó importantes hallazgos relacionados con las riñas y el sexo inducido, lo que puede evidenciar un alto grado de perturbación física y mental de manera permanente en la vida del individuo. No obstante, a pesar de estas consecuencias, más de la mitad de los encuestados se considera un tomador social, sin que esto afecte su calidad de vida. En este sentido, es claro que en la adolescencia las experiencias y vínculos que el individuo establece con el contexto familiar y con el medio externo al hogar influyen en el establecimiento del sentido de identidad, es decir, durante la adolescencia y juventud, además de aprender a establecer nuevos vínculos de amistad y de romance, los jóvenes necesitan llegar a reconocerse como personas únicas diferentes de los demás, integrar el concepto que tienen de sí mismos y fortalecer su autoestima $^{13,14}$.

En cuanto a las relaciones del consumo con las variables sociodemográficas, los jóvenes de procedencia urbana mostraron mayor probabilidad de consumo de bebidas alcohólicas, lo que puede explicarse por la diferencia del mundo social del joven de zonas rurales, en el cual la educación en valores es diferente, educado en la mayoría de los casos dentro de un contexto social y familiar de reciprocidad, diferente al estilo de vida del joven de ciudad, el cual llega a una institución universitaria y se adapta fácilmente a su nuevo estilo de vida, donde los grupos sociales para reconocerse utilizan el hábito del alcohol como una forma de bienestar y roce con las nuevas amistades, lo que ocasiona que se aumente la probabilidad del consumo ${ }^{15}$. Por otro lado, al ajustar los estimadores por edad, se presentan cambios importantes en la fuera estadística de las variables procedencia y género, lo que se puede explicar por la influencia que ejerce la poca autonomía que tienen los estudiantes menores de 18 años para consumir bebidas alcohólicas. En este mismo sentido, los estimadores de asociación para procedencia se aumentaron casi dos veces al ajustar por género, lo que puede explicarse por los prejuicios que desde el contexto familiar influyen en las mujeres para evitar convertirse en consumidoras habituales de alcohol desde sus primeros años de adolescencia.

Dentro de las implicaciones prácticas de los resultados, se asume que las bebidas alcohólicas para el grupo de estudiantes objeto se consideran una plataforma de interacción social, indicando que su patrón de consumo es indiscriminado para cualquier periodo del año e indiferente ante sus responsabilidades académicas, lo que puede ayudar a analizar el rendimiento académico de los estudiantes que consumen bebidas alcohólicas y compararlo con aquellos que no las consumen, para de esta forma tomar medidas preventivas que contribuyan a fortalecer la formación integral de los estudiantes.

Teniendo en cuenta las limitaciones del presente estudio, se puede concluir que la población estudiantil de la facultad de Odontología de la institución universitaria objeto de estudio presenta un alto consumo de bebidas alcohólicas, utilizando este hábito como mediador social entre su realidad y los eventos estresantes que la rodean, lo que puede ser una oportunidad para implementar medidas preventivas que ayuden a mejorar la calidad de vida académica y personal de los individuos.

\section{AGRADECIMIENTOS}

A la Universidad de Cartagena por su apoyo administrativo y financiero durante el desarrollo de este proyecto.

\section{BIBLIOGRAFÍA}

1. Vargas E, Barrera F. Adolescencia, relaciones románticas y actividad Sexual: una Revisión. Revista Colombiana de Psicología. 2002; 11:115-34. 
2. Abreu M, Fernández M, García M, Ángel A. Comportamiento del consumo de bebidas alcohólicas en estudiantes de medicina. Rev Cub Salud Pública. 1995; 21:6-8.

3. Frank E, Elon L, Naimi T, Brewer R. Alcohol consumption and alcohol counselling behaviour among US medical students: cohort study. BMJ. 2008; 337:21-55.

4. Baer JS. Student factors: understanding individual variation in college drinking. J Stud Alcohol Supp. 2002; 14:40-53.

5. Matute RC, Pillon SC. Alcohol consumption by nursing students in Honduras. Rev Lat Am Enfermagem. 2008; 16(5):84-9.

6. Alvarez ML. Nursing students' premature pregnancy and consumption of alcohol. Rev Lat Am Enfermagem. 2008; 16(5):77-83.

7. Bewick BM, Mulhern B, Barkham M, Trusler K, Hill AJ, Stiles WB. Changes in undergraduate student alcohol consumption as they progress through university. BMC Public Health. 2008; 19(8):163.

8. Geshi M, Hirokawa K, Taniguchi T, Fujii Y, Kawakami N. Effects of alcohol-related health education on alcohol and drinking behavior awareness among Japanese junior college students: a randomized controlled trial. Acta Med Okayama. 2007; 61(6):345-54.

9. Normas científicas, técnicas y administrativas para la investigación en salud. Resolución 008430 Ministerio de Salud República de Colombia; 1993.
10. Guibert W, Gutiérrez I, Martínez C. Comportamiento ante el alcohol de los estudiantes en las etapas de la adolescencia. Rev Cubana Med Gen Integr. 2005; 21:1-2.

11. Medina M, Natera G, Borges G, Cravioto P, Fleiz C, Tapia R. Del Siglo XX al tercer milenio, las adicciones y la salud pública: Drogas, Alcohol y Sociedad. Salud Mental. 2001; 24(4):3-19.

12. Herrán $\mathrm{O}$, Ardila M. Consumo de alcohol, riesgo de alcoholismo y alcoholismo en Bucaramanga, Colombia, 20022003. Colomb Med. 2005; 36(3):158-67.

13. Pinsky I, El jundi SA. Alcohol advertising and alcohol consumption among youngsters: review of the international literature. Rev Bras Psiquiatr 2008; 30(4):362-74.

14. Kaminer Y, Burleson JA, Burke RH. Efficacy of outpatient aftercare for adolescents with alcohol use disorders: a randomized controlled study. J Am Acad Child Adolesc Psychiatry. 2008; 47(12);1405-12.

15. Vendrame A, Pinsky I, Faria R, Silva R. Brazilian teenagers and beer advertising: relationship between exposure, positive response, and alcohol consumption. Cad Saude Publica. 2009; 25(2);359-65. 